\title{
EDITORIAL
}

\section{EL IMPACTO DEL CALOR EXTREMO EN LA SALUD: NUEVOS RETOS PARA LA EPIDEMIOLOGÍA Y LA SALUD PÚBLICA}

\author{
Ferran Ballester \\ Unidad de Epidemiología y Estadística. Escuela Valenciana de Estudios para la Salud. \\ CIBER de Epidemiología y Salud Pública (CIBERESP)
}

El pasado 7 de abril de 2008 se celebró la Jornada Mundial de la Salud organizada por la Organización Mundial de la Salud (OMS). Este año la OMS ha aprovechado la Jornada para lanzar una campaña dirigida a proteger la salud de los peligros del cambio climático ${ }^{1}$.

Según recoge el IV Informe del Panel Intergubernamental del Cambio Climático (IPCC) la temperatura de la Tierra ha aumentado $0,74^{\circ} \mathrm{C}$ en el periodo 1906 $2005^{2}$ [Intervalo de incertidumbre del $90 \%$ : $0,56-0,92]$. Además se tiene constancia de que este aumento no ha sido lineal en los últimos 50 años el incremento ha sido el doble que en la primera mitad del siglo XX. Once de los últimos 12 años (1995 -2006) se encuentran entre los 12 más calurosos desde que se registra la temperatura de la superficie de la Tierra (1850). Es decir, se observa una aceleración en el incremento de temperatura, que será mayor o menor según el escenario de emisiones futuras en que nos situemos.

En España un numeroso grupo de investigadores ha elaborado un informe sobre los impactos previsibles del cambio

Correspondencia:

Escuela Valenciana de Estudios en Salud (EVES)

C/Juan de Garay 21

46017 Valencia

Correo electrónico: ballester_fer@gva.es climático a instancias del Ministerio de Medio Ambiente ${ }^{3}$. En él se recogen como previsiones para la Península Ibérica un incremento térmico uniforme a lo largo del siglo XXI, con una tendencia media de aumento de $0,4{ }^{\circ} \mathrm{C}$ por década en invierno y de $0,7{ }^{\circ} \mathrm{C}$ por década en verano para el escenario menos favorable, y de $0,4^{\circ} \mathrm{C}$ y $0,6{ }^{\circ} \mathrm{C}$ por década, respectivamente, para el escenario más favorable. Este incremento de temperatura en promedio se puede asociar además con una mayor variabilidad en los fenómenos meteorológicos, por lo que se espera que las olas de calor sean más frecuentes, más intensas y de mayor duración.

Un ejemplo, claro y cercano, del impacto de las temperaturas extremas sobre la salud de la población lo tenemos en la ola de calor que azotó Europa en el verano de 2003. En su último informe, el Comité Ejecutivo de la OMS estima en más de 44.000 las defunciones en exceso en Europa a causa de las altas temperaturas ${ }^{4}$. En España, el trabajo llevado a cabo por el Centro Nacional de Epidemiología, estimó que más de 6.000 defunciones prematuras podrían ser atribuidas a las altas temperaturas del verano de $2003^{5}$. Desde ese año, las olas de calor son consideradas como crisis sanitarias y sociales y se han establecido sistemas de información y de alerta sobre posibles extremos térmicos, con 
consejos a la población de las medidas más importantes a tomar frente a dichas situaciones. Además, las Comunidades Autónomas, dentro de sus competencias, han puesto en marcha programas de prevención de los efectos del calor, a través de sus servicios sanitarios y sociales. Sin embargo, el desarrollo de sistemas de vigilancia en salud pública en relación a los extremos térmicos es escaso y puede ser poco eficaz para la prevención de su impacto en salud.

\section{SISTEMAS DE ALERTA Y VIGILANCIA DE LOS EFECTOS DEL CALOR}

Un número importante de países europeos ha puesto en marcha sistemas de control y alerta en relación al calor extremo. Francia e Italia son dos de los países donde el sistema ha tenido un desarrollo mayor $^{6,7}$. Su objetivo es permitir una respuesta adecuada de los servicios públicos, así como difundir medidas preventivas mediante consejos adecuados a las poblaciones vulnerables. En el año 2004 el Gobierno español puso en marcha el "Plan de acciones preventivas contra los efectos del exceso de temperaturas sobre la salud" que después fue mejorado en el año 2005. (http://www.msc.es/ciudadanos/saludAmbLaboral/planAltasTemp/2007/home.htm). En dicho Plan, junto a medidas de promoción y prevención, se estableció un sistema de alerta meteorológica a partir del percentil 95 de de las temperaturas medias medidas en cada provincia en los últimos 25 años y un sistema de vigilancia de la mortalidad diaria, del que se conoce poco respecto a su aplicabilidad en la práctica de la salud pública.

Para ser razonablemente eficaz un sistema de alerta debe estar basado en los determinantes del impacto en salud que, al menos en magnitud, pueden ser distintos en cada lugar. Es decir, es muy importante tener un conocimiento epidemiológico adecuado de la relación de la temperatura sobre la salud teniendo en cuenta las diferentes características climáticas, ambientales, demográficas, culturales y sociales de las poblaciones a estudio. En el ámbito europeo, el proyecto $\mathrm{PHEWE}^{8}$ evalúa la asociación entre factores meteorológicos y efectos agudos en salud (mortalidad, ingresos hospitalarios) en 16 ciudades europeas, entre las que se encuentran Barcelona y Valencia. El proyecto PHEWE ofrece la oportunidad de investigar la relación entre temperatura y mortalidad en una muestra amplia de ciudades europeas.

En España se dispone de un número importante de estudios sobre la relación de temperatura con la mortalidad, principalmente en Madrid, Barcelona, Valencia y Sevilla. En conjunto estos estudios indican que la relación entre la temperatura y la mortalidad tiene una forma propia en cada ciudad. Para el caso del impacto del calor, la magnitud de la relación y la temperatura a la cual el impacto es mayor (umbral) pueden ser distintas. En Madrid ${ }^{9}$ y Sevilla ${ }^{10}$ el aumento de la mortalidad ocurre abruptamente, especialmente en personas mayores, a partir de 36,5 y $41^{\circ} \mathrm{C}$ de temperatura máxima, respectivamente; mientras que en Barcelona $^{11}$ y Valencia ${ }^{12}$, con un clima suavizado por la proximidad del mar, el incremento de la mortalidad es menos marcado pero empieza antes, alrededor de los $29^{\circ} \mathrm{C}$ de temperatura máxima en Barcelona y a $24{ }^{\circ} \mathrm{C}$ de temperatura media en Valencia. Los diferentes periodos a estudio y algunas diferencias metodológicas dificultan la comparación de resultados de estos trabajos. Un estudio que incluyera diferentes ciudades españolas representativas de los diferentes climas y condiciones sociodemográficas, con datos más recientes, y en el que se utilizara metodología estándar común sería de gran utilidad a la hora de diseñar programas de prevención y sistemas de alerta y vigilancia en relación con el calor. 


\section{NECESIDADES DE INVESTIGACIÓN Y ACCIONES PARA PREVENIR EL IMPACTO DEL CALOR EXTREMO}

Para prevenir de manera efectiva el impacto del calor en las poblaciones más vulnerables, es fundamental tener en cuenta los factores demográficos y sociales que determinan la asociación con las altas temperaturas. Esta información permite dirigir los esfuerzos de la protección a los grupos de más riesgo. Los estudios epidemiológicos que han abordado este tema encuentran que son los ancianos y los niños pequeños, las mujeres, y los grupos de nivel socioeconómico más bajo, los grupos que sufren un mayor exceso de mortalidad durante las olas de calor ${ }^{13,14}$

Hasta ahora la mayoría de estudios han evaluado el impacto del calor sobre la mortalidad y existen pocos trabajos que evalúen el impacto sobre la morbilidad. El incremento de la morbilidad podría ser un indicador útil para la puesta en marcha de acciones tempranas que no supusieran irreversibilidad (la muerte) y, de esa manera, orientar la vigilancia más hacia la prevención de defunciones y enfermedades que a la descripción de la distribución y factores determinantes de la mortalidad. Entre los pocos trabajos existentes, se encuentra un estudio muy interesante realizado en Madrid por Cristina Linares y Julio Díaz ${ }^{15}$ en el que se describe que la asociación de los ingresos hospitalarios con las temperaturas extremas es de menor magnitud que la relación con la mortalidad, debido principalmente a que en los ingresos por enfermedades circulatorias no se aprecia una relación clara con la temperatura, mientras que para la mortalidad es la causa principalmente afectada . Estos resultados son similares a los observados por Kovats y cols. en Londres ${ }^{16}$ y a los del proyecto PHEWE (Michelozzi, comunicación personal), y podrían explicarse por la respuesta aguda del sistema cardiocirculatorio ante las altas temperaturas, pudiendo desencadenar un desenlace fatal antes de que el sujeto afectado pueda ingresar en el hospital.

Desde el punto de vista de la acción preventiva, el problema fundamental con los sistemas de vigilancia basados en la mortalidad es el retraso en obtener información en tiempo oportuno. Por ello se ha propuesto el uso de la información de las urgencias hospitalarias como indicador temprano de los efectos agudos de las olas de calor. En este número de la Revista Española de Salud Pública aparece un trabajo de García-Pina y colaboradores ${ }^{17}$ en el que se evalúa el efecto de la temperatura sobre el número de urgencias hospitalarias totales en la Región de Murcia. El fin principal del estudio es valorar la utilidad del número de urgencias y de los casos de golpes de calor en la vigilancia de la morbilidad debida a temperaturas elevadas. Los autores del trabajo concluyen que el número de urgencias totales no parece un indicador adecuado para monitorizar el efecto de la temperatura. Para conseguir dicho objetivo se necesitaría, como mínimo, información sobre el diagnóstico y la edad. Además la información se debería obtener de manera automática, y procesar e interpretar en tiempo oportuno para poder intervenir eficazmente.

Para solucionar dichos problemas se han propuesto algunas alternativas. En el Reino Unido se está desarrollando un trabajo conjunto entre la agencia de protección de la salud y el Sistema Nacional de Salud con el fin de explorar si un sistema de vigilancia sindrómica podría ser de utilidad para la detección de los efectos tempranos de las temperaturas elevadas ${ }^{18}$. La vigilancia sindrómica $^{19,20}$ se ha desarrollado para la detección de brotes epidémicos empleando técnicas automatizadas de obtención y procesamiento de datos de salud en tiempo real (o cercano al tiempo real) con el objetivo de detectar el brote más rápidamente que los métodos habituales empleados en salud 
pública. La vigilancia sindrómica ofrece así una oportunidad de detección temprana, pero atención, no sustituye a la vigilancia establecida en salud pública.

En Francia, un Hospital de París llevó a cabo un estudio para explorar la utilidad de un índice para la vigilancia en tiempo real de tres indicadores obtenidos, de manera automática en el servicios de urgencias: el porcentaje de pacientes mayores de 70 años, el porcentaje de pacientes con temperatura por encima de $39^{\circ} \mathrm{C}$ y, por último, el porcentaje de pacientes ingresados o fallecidos en el servicio de urgencias ${ }^{21}$. Los resultados del estudio sugieren que la información generada en el servicio de urgencias podría ser de utilidad para la detección temprana del impacto de el calor y, por tanto, para una intervención oportuna y eficaz.

Mientras estas nuevas aproximaciones son validadas y se va disponiendo de mayor evidencia epidemiológica, el papel fundamental de la salud pública debe ser la protección de la salud de la población, utilizando los métodos más eficaces y oportunos de que se disponga. Los sistemas de alerta meteorológica temprana basados en los estudios de relación con la mortalidad, la comunicación del riesgo a la población y la actuación preventiva de los servicios sanitarios y sociales, en un ámbito de coordinación intersectorial, son los métodos más utilizados a pesar de no disponer, especialmente para poblaciones vulnerables, de pruebas rotundas de su efectivi$\mathrm{dad}^{22}$. En el futuro se habrán de desarrollar otras medidas, como sistemas más sensibles y oportunos de vigilancia y de predicción meteorológica, pero también, como proponen Kovats y Hajat en su reciente revisión sobre el tema ${ }^{22}$, intervenciones que incluyan mejoras en las viviendas y en el diseño urbano de las ciudades, el manejo de las enfermedades crónicas y los cuidados de las personas más vulnerables, entre ellas los sujetos ancianos. Ante un fenómeno ambiental cambiante que afecta a todo el mundo, hace falta más investigación (epidemiológica y evaluativa), desarrollo de sistemas de vigilancia y alerta, e intervenciones en salud pública (en el ámbito individual, comunitario y global) con el fin de prevenir el impacto del calor extremo sobre la salud de la población. Nuevos retos para los profesionales de la epidemiología y la salud pública del presente y del futuro.

\section{BIBLIOGRAFÍA}

1. OMS. Día Mundial de la Salud 2008: proteger la salud frente al cambio climático. Disponible en: http://www.who.int/world-health-day/es

2. Intergovernmental Panel on Climate Change (IPCC). Climate Change 2007: The Physical Science Basis. Summary for Policymakers. Paris, release: 02/02/07. Disponible en: http://www.ipcc.ch/

3. Moreno JM (Ed.) 2005. Evaluación Preliminar General de los Impactos en España por Efecto del Cambio Climático. Madrid: Ministerio de Medio Ambiente; 2005. Disponible en http://www.mma.es/oecc.

4. WHO Executive Board, 16/01/2008. Climate change and health. Report by the Secretariat. Disponible en http://www.who.int/gb/ebwha/pdf_files/EB122/B12 2_4-en.pdf

5. Martínez F, Simón-Soria F, López-Abente G. Valoración del impacto de la ola de calor de 2003 sobre la mortalidad. Gac Sanit. 2004; 18:250-8

6. Pascal M, Laaidi K, Ledrans M, Baffert E, Caserio-Schönemann $\mathrm{C}$, Le Tertre A, et al. France's heat health watch warning system. Int J Biometeorol. 2006 Jan;50(3):144-53.

7. Michelozzi P, De Sario M, Accetta G, de'Donato F, Kirchmayer U, D'Ovidio M, Perucci CA; HHWWS Collaborative Group. Temperature and summer mortality: geographical and temporal variations in four Italian cities. J Epidemiol Community Health. 2006 May;60(5):417-23.

8. Michelozzi P, Kirchmayer U, Katsouyanni K, Biggeri A, McGregor G, Menne B, et al. Assessment and prevention of acute health effects of weather conditions in Europe, the PHEWE project: background, objectives, design. Environ Health. 2007 Apr 24; 6:12. 
9. Díaz J, Jordán A, García R, López C, Alberdi JC, Hernández E, et al. Heat waves in Madrid 19861997: effects on the health of the elderly. Int Arch Occup Environ Health. 2002 Mar; 75(3):163-70.

10. Díaz J, García R, Velázquez de Castro F, Hernández E, López C, Otero A. Effects of extremely hot days on people older than 65 years in Seville (Spain) from 1986 to 1997. Int J Biometeorol. 2002 Aug; 46(3):145-9.

11. Saez M, Sunyer J, Castellsagué J, Murillo C, Antó JM. Relationship between weather temperature and mortality: a time series analysis approach in Barcelona. Int J Epidemiol. 1995 Jun; 24(3): $576-82$.

12. Ballester F, Corella D, Pérez-Hoyos S, Sáez M, Hervás A. Mortality as a function of temperature. A study in Valencia, Spain, 1991-1993. Int J Epidemiol. 1997 Jun; 26(3):551-61.

13. Michelozzi P, de' Donato F, Bisanti L, Russo A, Cadum E, DeMaria M, et al. The impact of the summer 2003 heat waves on mortality in four Italian cities. Eur Surveill. 2005;10:7-8.

14. Borrell C, Marí-Dell'Olmo M, Rodríguez-Sanz M, Garcia-Olalla P, Caylà JA, Benach J, et al. Socioeconomic position and excess mortality during the heat wave of 2003 in Barcelona. Eur J Epidemiol. 2006; 21(9):633-40.

15. Linares C, Díaz J. Impact of high temperatures on hospital admissions: comparative analysis with previous studies about mortality (Madrid). Eur J Public Health. 2007 Nov 28; [Epub ahead of print]
16. Kovats RS, Hajat S, Wilkinson P. Contrasting patterns of mortality and hospital admissions during the hot weather and heat waves in Greater London, UK. Occup Environ Med. 2004;61:893-8.

17. García-Pina R, Tobías A, Sanz J, Navarro C, García-Fulgueiras A. Efecto del calor sobre el número de urgencias hospitalarias en la Región de Murcia durante los veranos del período 2000-2005 y su uso en la vigilancia epidemiológica. Rev Esp Salud Pública. 2008; 82: 153-66.

18. Menne B. Extreme Weather Events: What can We do to Prevent Health Impacts. En: Kisch W, Menne B, Bertollini R (eds). Extreme Weather Events and Public Health Responses. Berlin: Springer. WHO Europe; 2007. p. 265-271.

19. Kelly J. Henning . Overview of Syndromic Surveillance. What is Syndromic Surveillance? MMWR Morb Mortal Wkly Rep 53 (Suppl) 5-11.

20. Moore KM, Edgar BL, McGuinness D. Implementation of an automated, real-time public health surveillance system linking emergency departments and health units: rationale and methodology. CJEM. 2008;10(2):114-9

21. How emergency departments might alert for prehospital heat-related excess mortality? Claessens YE, Taupin P, Kierzek G, Pourriat JL, Baud M, Ginsburg C, et al. Critical Care. 2006, 10:R156 (doi:10.1186/cc5092)

22. R. Sari Kovats and Shakoor Hajat Heat Stress and Public Health: A Critical Review Annu Rev Public Health. 2008. 29:41-55. 